\title{
ANÁLISE DA EXECUÇÃO DE UM ALGORITMO NUMÉRICO CBS PARA SIMULAÇÃO DE ESCOAMENTO UTILIZANDO ELEMENTOS FINITOS
}

\section{ANALYSIS OF CBS NUMERICAL ALGORITHM EXECUTION TO FLOW SIMULATION USING THE FINITE ELEMENT METHOD}

\author{
Marcos Paulo de Carvalho Oliveira $^{1} \quad$ Vicente Luiz Scalon $^{1} \quad$ Alcides Padilha $^{1}$ \\ Recibido 17 de julio de 2007, aceptado 27 de abril de 2009 \\ Received: July 17, 2007 Accepted: April 27, 2009
}

\begin{abstract}
RESUMO
Neste trabalho é realizada uma análise da aplicação e do tempo de execução de um algoritmo numérico desenvolvido para a simulação de escoamento isotérmico de fluido incompressível. No código, emprega-se o algoritmo denominado esquema baseado nas características (CBS-characteristic based split), em que é usado o acoplamento da compressibilidade artificial (AC) para as equações da pressão e velocidades. A discretização foi realizada com o método dos elementos finitos, empregando-se uma malha de elementos bilineares. O programa GNU-Octave foi utilizado para implementar e executar as rotinas. Por meio do problema clássico da cavidade recirculante, foram avaliados resultados para diversos valores do número de Reynolds. Esses resultados mostraram boa concordância quando comparados aos disponíveis na literatura. A análise do tempo de execução das rotinas mostrou ainda que a montagem das matrizes é a etapa que exige maior consumo de tempo da implementação.
\end{abstract}

Palavras-chave: CBS, compressibilidade artificial, método de elementos finitos, cavidade recirculante, GNU-Octave.

\section{ABSTRACT}

This work intends to analyze the application and execution time of a numerical algorithm that simulates incompressible and isothermal flows. It was used the explicit scheme of the "Characteristic Based Split" (CBS) algorithm and the "Artificial Compressibility" (AC) scheme for coupling pressure-velocity equations. The discretization was done with the finite element method using a bilinear elements grid. The free software GNU-Octave was used for implementation and execution of routines. The results were analyzed using the classic lid-driven cavity problem. This work shows results for tests with several Reynolds' number. The results for these tests show a good agreement when compared with previous ones obtained from bibliography. The code runtime's analysis shows yet that the matrix's assembly is the part of greater consumption time in the implementation.

Keywords: CBS, artificial compressibility, finite element method, lid-driven cavity, GNU-Octave.

\section{INTRODUÇÃO}

A simulação numérica, ou modelagem computacional, em mecânica de fluídos e transferência de calor teve um grande crescimento nos últimos 30 anos, fruto principalmente dos avanços dos computadores e das técnicas de solução. Com isso, a simulação numérica constitui-se em uma ferramenta poderosa para a solução de problemas em diversas áreas, inclusive nas engenharias. A versatilidade e a relativa simplicidade de aplicação desses métodos implicaram em numa sensível diminuição dos trabalhos de laboratórios e, conseqüentemente, dos seus custos.
Por conta disso, essa área está em franca expansão, com aplicação no desenvolvimento de produtos industriais, e pesquisas científicas básicas e aplicadas.

Entretanto, sua aplicação a um problema depende, na maioria das vezes, da solução de equações diferenciais que o representam. Em problemas bi e tridimensionais, esse procedimento depende da solução de equações diferenciais parciais onde são comumente utilizados os métodos de elementos finitos, das diferenças finitas ou dos volumes finitos. A escolha entre essas técnicas depende muito mais do conhecimento prévio que da

1 Universidade Estadual Paulista Júlio de Mesquita Filho - UNESP. Bauru, SP, Brasil. E-mail: mpcoliveira@ feb.unesp.br, scalon@ feb.unesp.br, padilha@feb.unesp.br 
eficiência na solução de um problema específico. Para cada uma dessas técnicas, existe ainda uma série de algoritmos e programas que podem ser utilizados na análise numérica. No caso específico do método dos elementos finitos, é comum o uso tanto do método das características como a solução acoplada por meio do esquema da compressibilidade artificial. Embora essas técnicas sejam conhecidas há algum tempo, somente recentemente o seu uso teve um aumento significativo, principalmente a partir do trabalho apresentado por Massaroti, Nithiarasu e Zienkiewicz [1]. Este trabalho mostrou uma série de soluções que apresentavam boa concordância com diversos estudos experimentais e teóricos realizados anteriormente. Dessa forma, os autores já o consideravam um método muito promissor e com boas possibilidades de utilização em problemas mais complexos.

Dando sequiência ao desenvolvimento do modelo, Zienkiewicz, Nithiarasu, Codina, Vazquez e Ortiz [2] apresentam inovações que permitiam substituir o método de Taylor-Galerkin (ou Lax-Wendroff), utilizados para solução de escoamentos compressíveis. Esse novo algoritmo, então denominado CBS, foi aplicado para uma grande variedade de situações, incluindo escoamentos completamente incompressíveis e escoamentos com superfície aberta.

Fazendo uma compilação dos trabalhos anteriormente publicados, Zienkiewicz e Taylor publicam um livro com a descrição completa da metodologia CBS [3]. Utilizando-se da discretização de Galerkin e dos métodos de elementos finitos, os autores mostram os passos da resolução, as formulações utilizadas e avaliações do método, entre outras informações. Essa metodologia, a partir desse seu maior detalhamento, serviu como base de desenvolvimento para diversas pesquisas no mundo inteiro, que contribuíram para divulgar o novo modelo.

Desse mesmo período, destaca-se, ainda, outro trabalho de Nithiarasu e Zienkiewicz no qual são apresentadas as técnicas de estabilização com os passos de tempo [4]. Neste caso, o principal foco residia nos escoamentos incompressíveis e na aceleração convectiva, utilizando o algoritmo CBS. Os modelos foram novamente testados para o caso da cavidade recirculante e, assim, foi possível uma avaliação dos resultados obtidos com a nova técnica. Existem diversos outros artigos disponíveis na literatura que se utilizam do mesmo método e com excelentes resultados, cabendo destacar os trabalhos [5-9].

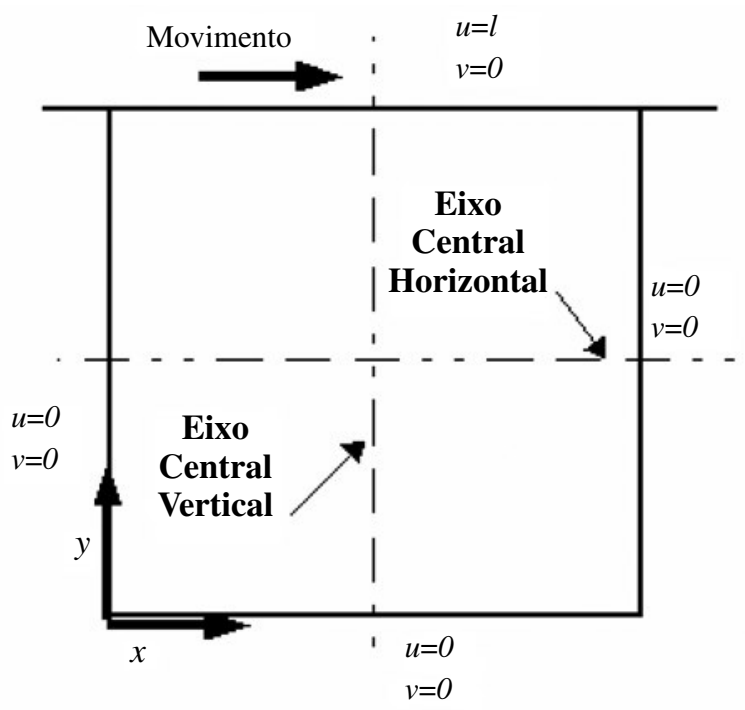

Figura 1. Cavidade Recirculante: condições de contorno e linhas auxiliares.

Para todos os modelos apresentados, é commun a solução de um problema clássico para testes de estabilidade: a cavidade recirculante ou Lid-driven cavity. A figura 1 ilustra um esquema desse problema, que vem sendo usado há tempos na verificação de modelos numéricos. Ele consiste numa cavidade fechada na qual a face superior se desloca com velocidade uniforme e todas as demais paredes permanecem estáticas. Uma solução clássica para o problema, com resultados numéricos e experimentais, foi apresentada por U. Ghia, K.N. Ghia e Shim [10]. Neste trabalho podem ser encontrados valores de referência ideais para a comparação de resultados, o que justifica a sua larga utilização como padrão de soluções. Uma série de outros resultados para cavidade recirculante, com diferentes geometrias também foram obtidos por Erturk [11].

Neste trabalho, propõe-se uma implementação das rotinas do esquema CBS para solução do escoamento da cavidade recirculante num ambiente matemático. Para tanto, será utilizado o programa GNU-Octave, que é um programa livre, aberto e com distribuição gratuita e pode ser executado tanto nos sistema operacionais Linux e Windows [12]. Para a validação, serão realizados testes com o intuito de se verificar a precisão dos resultados obtidos. Por fim, será feita uma análise do tempo consumido na execução de uma simulação de escoamento isotérmico incompressível, em que se procura detectar os pontos críticos para o consumo de tempo no programa. 


\section{METODOLOGIA}

As formulações utilizadas neste trabalho tiveram como base, principalmente, os procedimentos descritos na referência [3], com pequenas alterações para o tratamento do problema adimensional e voltado para fluido newtoniano.

As equações de Navier-Stokes descrevem a solução do problema e são apresentadas, na sua forma adimensional nas equações (2), (3) e (4). Os adimensionais utilizados são obtidos a partir dos valores dimensionais $(+)$ na forma:

$$
\begin{aligned}
& \rho=\frac{\rho^{+}}{\rho_{r}}, \quad \mathrm{u}=\frac{\mathrm{u}^{+}}{\mathrm{U}^{+}}, \quad \mathrm{v}=\frac{\mathrm{v}^{+}}{\mathrm{U}^{+}}, \quad \mathrm{c}=\frac{\mathrm{c}^{+}}{U^{+}}, \quad g=\frac{g^{+}}{L U^{+2}} \\
& \mathrm{p}=\frac{\mathrm{p}^{+}}{\rho_{\mathrm{r}} U^{+2}}, \quad \mathrm{x}=\frac{\mathrm{x}^{+}}{L}, \quad \mathrm{y}=\frac{\mathrm{y}^{+}}{L}, \quad \operatorname{Re}=\frac{\rho_{r} U^{+} L}{\mu}
\end{aligned}
$$

\section{- Equação da conservação da massa:}

$$
\begin{aligned}
& \frac{\partial \rho}{\partial t}=-\frac{\partial \rho u}{\partial x}-\frac{\partial \rho v}{\partial y} \\
& \frac{1}{c^{2}} \frac{\partial p}{\partial t}=-\frac{\partial U}{\partial x}-\frac{\partial V}{\partial y}
\end{aligned}
$$

- Equações da conservação da quantidade de movimento nas direções $x$ e $y$ :

$$
\begin{gathered}
\frac{\partial(\rho u)}{\partial t}+\frac{\partial(\rho u u)}{\partial x}+\frac{\partial(\rho u v)}{\partial y}= \\
\frac{\partial(U)}{\partial t}+\frac{\partial(u U)}{\partial x}+\frac{\partial(v U)}{\partial y}= \\
\frac{1}{\operatorname{Re}}\left(\frac{\partial^{2} u}{\partial x^{2}}+\frac{\partial^{2} u}{\partial y^{2}}\right)-\frac{\partial p}{\partial x}-\rho g_{x} \\
\frac{\partial(\rho v)}{\partial t}+\frac{\partial(\rho v \mathrm{u})}{\partial x}+\frac{\partial(\rho \mathrm{v} v)}{\partial y}= \\
\frac{\partial(V)}{\partial t}+\frac{\partial(u V)}{\partial x}+\frac{\partial(\mathrm{v} V)}{\partial y}= \\
\frac{1}{\operatorname{Re}}\left(\frac{\partial^{2} \mathrm{v}}{\partial x^{2}}+\frac{\partial^{2} \mathrm{v}}{\partial y^{2}}\right)-\frac{\partial p}{\partial y}-\rho g_{y}
\end{gathered}
$$

\section{ALGORITMO CBS}

O método utilizado como base para este esquema foi o proposto originalmente por [3] com as pequenas modificações do algoritmo original. Todas as características essenciais da forma padronizada, completamente conservativa, podem ser escritas pelo conjunto de equações de Navier-Stokes apresentadas anteriormente nas equações (2) a (4).

As equações (3) a (4) podem ser discretizadas usando o processo de Galerkin exceto pelo termo transiente, no qual é utilizada uma aproximação de Euler. O método das características é uma alternativa aos tradicionais esquemas "upwind" normalmente utilizados em soluções de escoamento e leva ao aparecimento de um termo associado a $\Delta t^{2}$.

Duas alternativas de aproximações, chamadas de Split A e Split B, podem ser realizadas para o termo $Q$ [3]. A diferença básica entre esses dois esquemas consiste em que, no primeiro, são removidos todos os termos do gradiente de pressão da equação (5) e, no segundo, o termo do gradiente é mantido para o dado incremento de tempo. Como o algoritmo Split A será adotado neste trabalho, por ser considerado o mais estável.

$$
\begin{gathered}
U_{i}^{n+1}-\mathrm{U}_{\mathrm{i}}^{\mathrm{n}}= \\
-\Delta \mathrm{t}\left[\frac{\partial\left(u_{j} \mathrm{U}_{\mathrm{i}}\right)}{\partial x_{j}}-\frac{1}{\operatorname{Re}}\left(\frac{\partial^{2} \mathrm{u}_{\mathrm{i}}}{\partial x_{j}^{2}}\right)-Q^{n+\theta_{2}}+\rho g_{i}\right]_{(5)}^{n} \\
+\frac{\Delta \mathrm{t}^{2}}{2} U_{k}^{n} \frac{\partial}{\partial x_{k}}\left[\frac{\partial\left(u_{j} \mathrm{U}_{\mathrm{i}}\right)}{\partial x_{j}}-Q^{n+\theta_{2}}+\rho g_{i}\right]^{n}
\end{gathered}
$$

O Split A utiliza-se, para a solução do problema, de uma variável auxiliar $\Delta U * i$, tal que:

$$
\begin{aligned}
\Delta U_{i}^{* 1}= & U_{i}^{*}-\mathrm{U}_{\mathrm{i}}^{\mathrm{n}}= \\
\Delta \mathrm{t} & {\left[-\frac{\partial}{\partial x_{j}}\left(u_{j} \mathrm{U}_{\mathrm{i}}\right)+\frac{1}{\operatorname{Re}}\left(\frac{\partial^{2} \mathrm{u}_{\mathrm{i}}}{\partial x_{j}^{2}}\right)-\rho g_{i}\right]^{n} } \\
& \quad-\frac{\Delta \mathrm{t}^{2}}{2}\left[u_{k} \frac{\partial}{\partial x_{k}}\left(\frac{\partial}{\partial x_{j}}\left(u_{j} \mathrm{U}_{\mathrm{i}}\right)+\rho g_{i}\right)\right]^{n}
\end{aligned}
$$

A equação (6) será resolvida para um incremento de tempo explícito aplicado para a forma discretizada, o que torna possível uma solução. A seguinte correçao é avaliada primeiramente no incremento da pressão:

$$
\begin{aligned}
& \Delta U_{i}=U_{i}^{n+1}- \mathrm{U}_{\mathrm{i}}^{\mathrm{n}}= \\
& \Delta U_{i}^{*}-\Delta \mathrm{t} \frac{\partial \mathrm{p}^{\mathrm{n}+\theta_{2}}}{\partial x_{i}}-\frac{\Delta \mathrm{t}^{2}}{2} u_{k} \frac{\partial \mathrm{Q}_{\mathrm{i}}^{\mathrm{n}}}{\partial x_{k}}
\end{aligned}
$$

Da equação (2), utilizando-se um termo de estabilização numérica $\beta$ em lugar da velocidade de som "c" e cujos detalhes serão discutidos adiante, tem-se: 


$$
\begin{aligned}
\Delta \rho=\frac{1}{\beta^{2}} \Delta p= & -\Delta t\left[\frac{\partial U_{i}^{n}}{\partial x_{i}}+\theta_{1} \frac{\partial \Delta U_{i}^{*}}{\partial x_{i}}\right] \\
& +\Delta \mathrm{t}^{2} \theta_{1}\left[\frac{\partial^{2} p^{n}}{\partial x_{i}^{2}}+\theta_{2} \frac{\partial^{2} \Delta p}{\partial x_{i}^{2}}\right]
\end{aligned}
$$

em que $\Delta U^{*}{ }_{i}$ e os termos de pressão são calculados pela equação (6).

A solução deste sistema de equações por meio de elementos finitos depende ainda da sua discretização no espaço. Este procedimento é feito para os diversos casos utilizando de uma aproximação da função peso da de Galerkin. Utilizando-se esse esquema para a forma fraca da equação (6) tem-se:

$$
\begin{aligned}
& \int_{\Omega} N_{u}^{k} \Delta U_{i}^{*} \mathrm{~d} \Omega= \\
& +\Delta \mathrm{t}\left[-\int_{\Omega} N_{u}^{k} \frac{\partial}{\partial x_{j}}\left(u_{j} \mathrm{U}_{\mathrm{i}}\right) d \Omega\right]^{n} \\
& +\frac{\Delta t}{\operatorname{Re}}\left[\int_{\Omega} \frac{\partial N_{u}^{k}}{\partial x_{j}} \frac{\partial \mathrm{u}_{\mathrm{i}}}{\partial x_{j}} d \Omega\right]^{n} \\
& +\Delta \mathrm{t}\left[\int_{\Omega} N_{u}^{k} \rho g_{i} d \Omega\right]^{n} \\
& +\frac{\Delta \mathrm{t}^{2}}{2}\left[\int_{\Omega} \frac{\partial}{\partial x_{l}}\left(N_{u}^{k} u_{k}\right)\left(-\frac{\partial}{\partial x_{j}}\left(u_{j} \mathrm{U}_{\mathrm{i}}\right)\right) d \Omega\right]^{n} \\
& +\frac{\Delta \mathrm{t}^{2}}{2}\left[\int_{\Omega} \frac{\partial}{\partial x_{l}}\left(N_{u}^{k} u_{k}\right) \rho g_{i} d \Omega\right]^{n} \\
& +\Delta \mathrm{t}\left[\int_{\Gamma} N_{u}^{k} \tau_{i j} n_{j} d \Gamma\right]^{n}
\end{aligned}
$$

que após o uso da aproximação nodal, pode ser reescrita na forma matricial:

$$
\begin{aligned}
\Delta \tilde{U}_{i}^{*}=-M_{u}^{-1} \Delta \mathrm{t}\left(C_{u} u\right. & \left.+\frac{1}{\operatorname{Re}} K_{t} u-f\right) \\
& +M_{u}^{-1} \Delta \mathrm{t}\left(K_{u} \tilde{U}+f_{s}\right)
\end{aligned}
$$

A equação (10) representa o Passo 1 do esquema CBS, onde:

$$
\begin{aligned}
& M_{u}=\int_{\Omega} N_{u}^{T} N_{u} d \Omega, \\
& C_{u}=\int_{\Omega} N_{u}^{T}\left(\nabla\left(u N_{u}\right)\right) d \Omega, \\
& K_{t}=\int_{\Omega}\left(\nabla N_{u}\right)^{T} N_{u} d \Omega, \\
& K_{u}=-\frac{1}{2} \int_{\Omega}\left(\nabla^{T}\left(u N_{u}\right)\right)^{T}\left(\nabla^{T}\left(u N_{u}\right)\right) d \Omega, \\
& f=\int_{\Omega} \mathrm{N}_{\mathrm{u}}^{\mathrm{T}} \rho g d \Omega+\int_{\Gamma} \mathrm{N}_{u}^{\mathrm{T}} \mathrm{t}_{\mathrm{d}} \mathrm{d} \Gamma \mathrm{e} \\
& f_{s}=-\frac{1}{2} \int_{\Omega}\left(\nabla^{T}\left(u N_{u}\right)\right)^{T} \rho g d \Omega,
\end{aligned}
$$

A partir da forma fraca da equação (7) utilizando-se uma função peso da aproximação de Galerkin, tem-se:

$$
\begin{aligned}
\int_{\Omega} N_{p}^{k} \Delta \rho d \Omega=\int_{\Omega} N_{p}^{k} \frac{1}{\beta^{2}} \Delta p d \Omega= \\
+\Delta t \int_{\Omega} \frac{\partial N_{p}^{k}}{\partial x_{i}}\left[U_{i}^{n}+\theta_{1} \Delta U_{i}^{*}-\Delta t \theta_{1} \frac{\partial p^{n+\theta_{2}}}{\partial x_{i}}\right] d \Omega \\
\quad-\Delta t \int_{\Gamma} N_{p}^{k}\left[U_{i}^{n}+\theta_{1} \Delta U_{i}^{*}+\Delta t \theta_{1} \frac{\partial p^{n+\theta_{2}}}{\partial x_{i}}\right] n_{j} d \Gamma
\end{aligned}
$$

Da mesma forma, a equação (12) pode ser escrita na forma matricial:

$$
\begin{aligned}
& \left(M_{p}+\Delta t^{2} \theta_{1} \theta_{2} H\right) \Delta \tilde{p}= \\
& \quad \Delta t\left(G \tilde{U}^{n}+\theta_{1} G \Delta \tilde{U}^{*}-\Delta t \theta_{1} H \tilde{p}^{n}-f_{p}\right)
\end{aligned}
$$

representando o Passo 2 do esquema CBS, onde:

$$
\begin{aligned}
& M_{p}=\int_{\Omega} N_{p}^{T}\left(\frac{1}{\beta^{2}}\right)^{n} N_{p} d \Omega \\
& H=\int_{\Omega}\left(\nabla N_{p}\right)^{T} \nabla N_{p} d \Omega \\
& G=\int_{\Omega}\left(\nabla N_{p}\right)^{T} N_{u} d \Omega \\
& \mathrm{f}_{\mathrm{p}}=\int_{\Gamma} N_{p}^{T} n^{T}\left[\tilde{U}^{n}+\theta_{1}\left(\tilde{U}^{*}-\Delta t \nabla p^{n+\theta_{2}}\right)\right] d \Gamma
\end{aligned}
$$

Calculando a forma fraca da equação (7) utilizando uma função peso da aproximação de Galerkin, tem-se:

$$
\begin{aligned}
\int_{\Omega} N_{p}^{k} \Delta U_{i} d \Omega & =\int_{\Omega} N_{p}^{k} \Delta U_{i}^{*} d \Omega \\
& -\Delta t \int_{\Omega} N_{p}^{k}\left(\frac{\partial p^{n}}{\partial x_{i}}+\theta_{2} \frac{\partial \Delta p}{\partial x_{i}}\right) d \Omega \\
+ & \frac{\Delta t^{2}}{2} \int_{\Omega} \frac{\partial}{\partial x_{k}}\left(N_{p}^{k} u_{k}\right)\left(\frac{\partial p^{n}}{\partial x_{i}}\right) d \Omega
\end{aligned}
$$

Novamente, a equação (15) pode ser escrita na forma matricial:

$$
\Delta \tilde{U}=\Delta \tilde{U}^{*}-M_{p}^{-1}\left[\Delta t G^{T}\left(p^{n}+\theta_{2} \Delta \tilde{p}\right)+\frac{\Delta t^{2}}{2} P \tilde{p}^{n}\right]
$$

que representa o Passo 3 do esquema CBS, onde:

$$
P=\int_{\Omega} \nabla\left(u N_{u}\right)^{T} \nabla N_{p} d \Omega
$$


o procedimento geral de solução, com a aplicação desses diversos passos, está apresentado no fluxograma da figura 2.

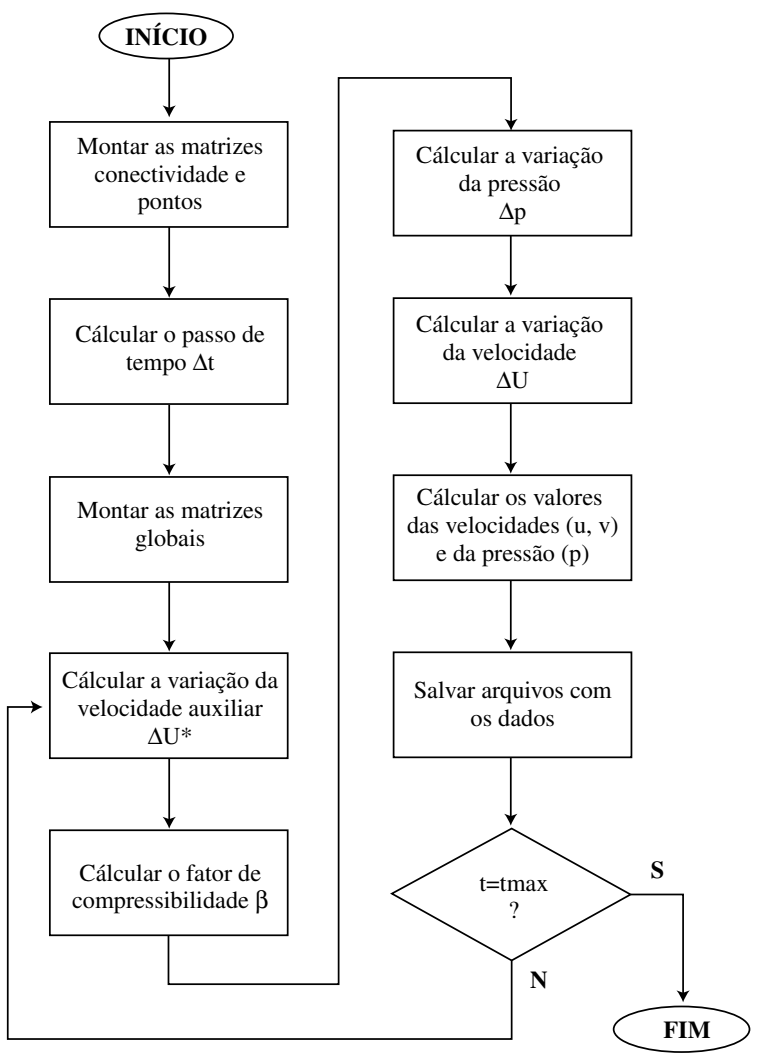

Figura 2. Fluxograma geral do processo de solução.

\section{COMPRESSIBILIDADE ARTIFICIAL}

O valor do fator da velocidade do som "c" utilizado na equação (2) pode acarretar em restrições na escolha do incremento de tempo. Desse modo, normalmente é empregado um parâmetro $\beta$ como um fator de compressibilidade artificial, que permite acelerar o processo de convergência para cada instante de tempo.

Este fator não está associado às propriedades físicas do fluido, mas aos diferentes números de Reynolds e também aos diferentes regimes (denominados convecção e difusão). Segundo Nithiarasu [13], o valor utilizado para a compressibilidade artificial pode ser calculado, conforme equação (18).

$$
\beta=\operatorname{máx}\left(\varepsilon, v_{c o n v}, v_{d i f f}\right)
$$

onde, $\varepsilon$ é uma constante igual $0,5, v_{\text {conv }}$ é a velocidade convectiva e $v_{\text {diff }}$ é a velocidade de difusão que podem ser calculados conforme segue:

$$
v_{\text {conv }}=\left(u_{i} u_{i}\right)^{1 / 2} \quad \text { e } \quad \mathrm{v}_{\text {diff }}=\frac{2}{h \operatorname{Re}}
$$

Sendo $h$ o valor do menor tamanho utilizado no elemento local.

\section{INCREMENTO DE TEMPO}

O incremento de tempo, um parâmetro muito importante em soluções, deve ser o maior possível de forma que não comprometa a estabilidade da solução e, também, diminua a quantidade de passos a serem utilizados para atingir a solução em regime permanente. $\mathrm{O}$ incremento de tempo para o método foi descrito em [3] e é calculado pelas seguintes etapas:

$$
\Delta t_{c}=\frac{h}{u-\beta}
$$

Ao valor obtido em (20) deve-se aplicar um fator de segurança que deve ser obtido por meio de tentativa, ou seja, considera-se o valor encontrado na execução da solução e verifica se ocorre a estabilidade da solução. Em caso negativo, o valor é multiplicado por um percentual deste número até que a estabilidade seja mantida. Neste trabalho os valores utilizados foram de 0,4 para $R e=100$ e 1.000 e 0,6 para $\mathrm{Re}=400$. Assim:

$$
\Delta t=F S * \Delta t_{c}
$$

onde $F S$ é o fator de segurança utilizado para garantir a convergência do modelo.

\section{RESULTADOS E DISCUSSÕES}

O procedimento descrito foi utilizado na montagem do código computacional e implementado sobre a plataforma matemática: o GNU-Octave [12]. Todas as subrotinas foram escritas na forma de funções que eram chamadas a partir do programa principal que segue a lógica apresentada na figura 2. O modelo de malha utilizada para a solução da cavidade recirculante com $\mathrm{Re}=100$ pode ser visto na figura 3. Para a solução do escoamento com números de Reynolds de 100 e 400, foi utilizada uma malha irregular de $21 \times 21$ e para Reynolds 1.000 , uma malha $41 \times 41$.

As malhas utilizadas foram utilizadas com uma deformação exponencial a partir da borda superior e das laterais de forma a obter um maior refinamento de malha onde se tem os maiores gradientes de velocidades. Este procedimento, normalmente, apresenta bons resultados em malhas mais grosseiras. 


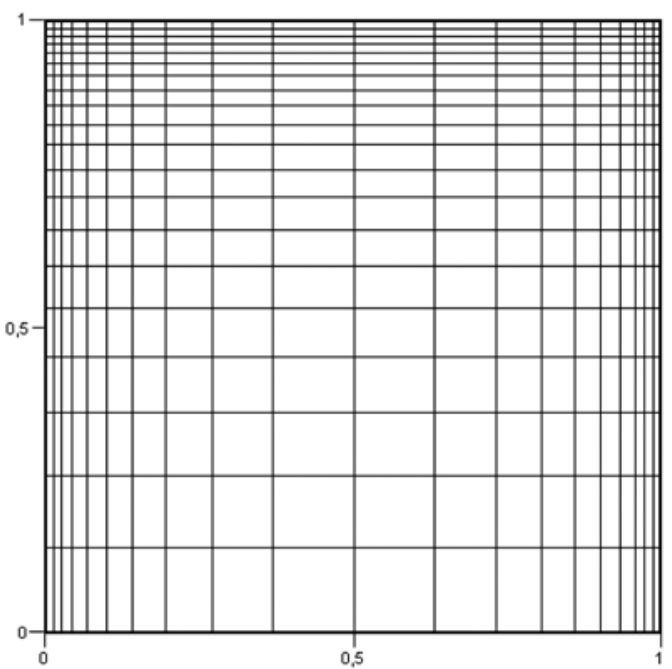

Figura 3. Malha Exponencial 21 x 21.

Para cada teste de Reynolds foi montado uma curva comparativa dos resultados mostrando os valores das velocidades obtidos nas linhas centrais da cavidade como mostrado na figura 1 .

Os resultados são apresentados na forma gráfica nas figuras 4 e 5, comparados com aqueles obtidos por U. Ghia, K.N. Ghia e Shin [10].

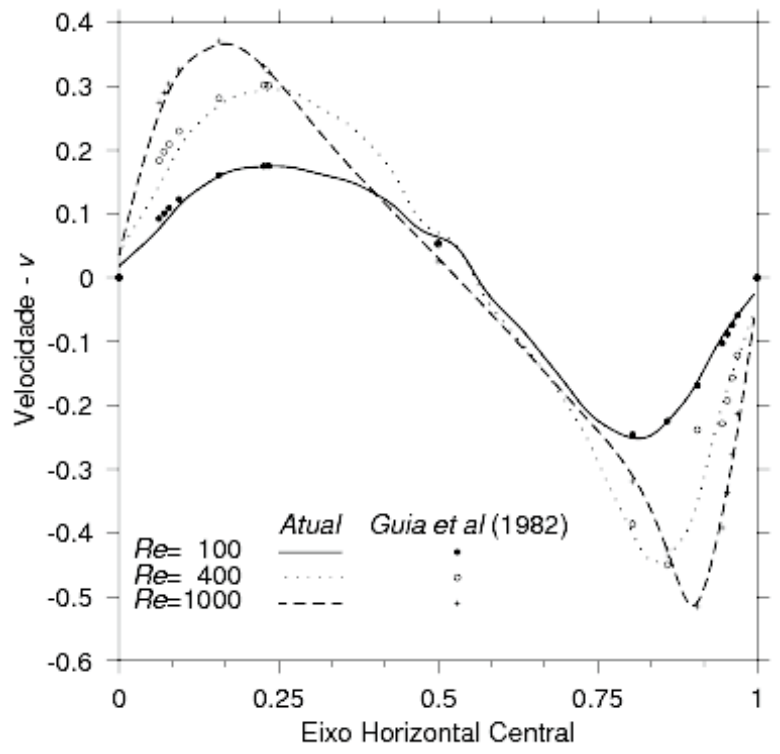

Figura 4. Comparação do perfil de velocidades verticais (v) no eixo central horizontal.

A figura 4 apresenta a distribuição de resultados para o perfil das velocidades verticais na linha central horizontal da cavidade. Os resultados para os diversos valores de $\mathrm{Re}=100$ a $\mathrm{Re}=1000$ foram comparados com aqueles apresentados na referência [10] permitindo verificar a boa precisão do modelo mesmo sem a utilização de malhas muito refinadas. Os valores de incremento de tempo e número de iterações necessárias variavam para cada regime do escoamento.

A figura 5, por sua vez, apresenta a distribuição para o perfil das velocidades horizontais na linha central vertical da cavidade. Novamente, os resultados foram comparados aos da referência [10] e foi verificado uma boa concordância entre os mesmos. Os resultados mostraram que, para este tipo de geometria, é muito mais fácil alcançar a concordância do perfil de velocidades horizontais que verticais.

Por outro lado, os resultados comprovam a eficácia da implementação e mostram que as pequenas alterações realizadas no modelo não prejudicaram a qualidade dos resultados.

Comprovada a eficácia do modelo pode-se discutir agora a sua implementação. Um dos aspectos críticos de qualquer modelo é o tempo necessário para a sua execução já que as respostas têm que ser obtidas num intervalo de tempo aceitável.

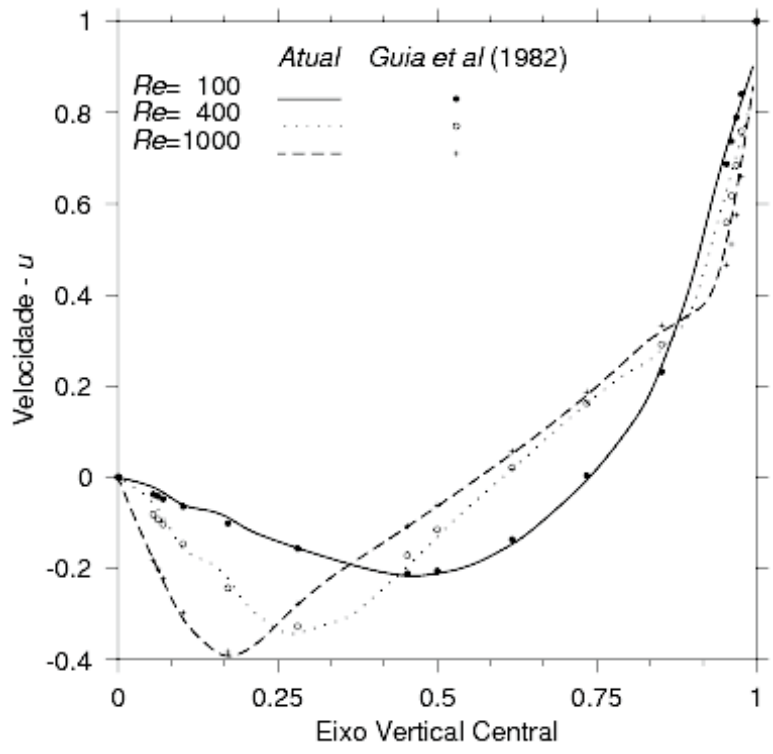

Figura 5. Comparação do perfil de velocidades hotizontais (u) no eixo central vertical.

Sendo assim foi feito um estudo do consumo de tempo nas diversas etapas do programa similares ao mostrado na figura 2. A figura 6 apresenta a distribuição do tempo consumido considerando apenas um passo de tempo. 
Os testes foram feitos para o caso específico do $\mathrm{Re}=100$ e malha ( $21 \times 21)$. O tempo apresentado se refere ao código rodando no Gnu-Octave versão 2.1.7.3, rodando em um computador Athlon XP $1900+$ com $256 \mathrm{Mb}$ com Linux Kernel 2.6.17.10.

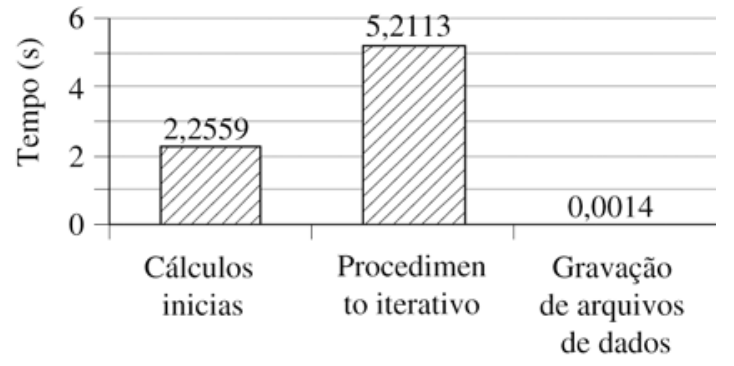

Figura 6. Tempos gastos em cada uma das etapas principais de execução do programa.

Para esta análise houve uma subdivisão do código em três partes, conforme se segue:

- cálculos inicias: compreende a divisão dos pontos que formam a malha com suas respectivas coordenadas, conectividades e condições de contorno; o cálculo do incremento de tempo e a montagem das matrizes auxiliares iniciais. Esta etapa é somente realizada uma vez durante a rotina.

- procedimento iterativo: compreende o cálculo da variação da velocidade auxiliar, a variação de pressão e posteriormente a variação da velocidade, montando suas respectivas matrizes e a solução das equações diferenciais apresentadas na metodologia deste presente trabalho. Esta etapa é realizada diversas vezes durante a rotina, dependendo da quantidade de passos necessários para a obtenção da estabilização dos resultados.

- gravação de arquivos de dados: compreende a gravação dos arquivos de dados para a geração dos gráficos e análises dos resultados. Por motivo de segurança e acompanhamento optou-se por atualizar os arquivos a cada 25 passos e gravação da cópia de análise a cada 1.000 passos para $\operatorname{Re}=100$.

Conforme se pode perceber da figura 6 , o procedimento iterativo é responsável pelo consumo da maior parcela de tempo, uma vez que abriga a maioria dos procedimentos mais complexos. Um complicador deste fato é que, justamente, este passo é o que precisa ser repetido por inúmeras vezes. Dessa forma, o número de passos multiplicado pelo tempo de cada iteração será o indicador fundamental do tempo necessário para a finalização da execução do programa.
Como se trata de um fator limitante será realizado um detalhamento acerca do consumo de tempo no procedimento iterativo. A distribuição do tempo consumido pode ser vista na figura 7, em que é feita uma subdivisão em outras três partes:

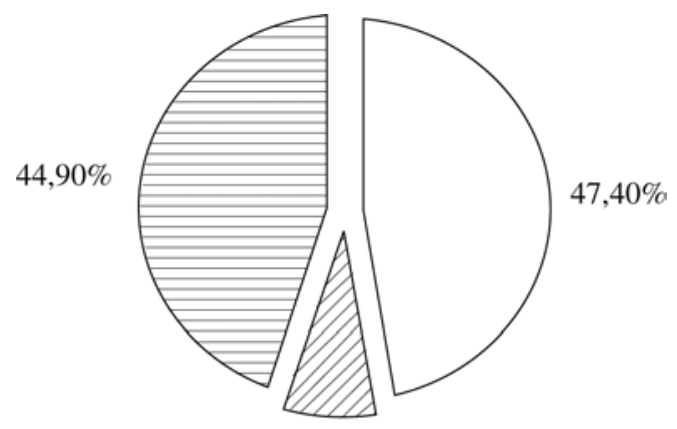

$7,70 \%$

\begin{tabular}{|ccc|}
\hline $\begin{array}{l}\text { Cálculo do } \\
\text { desvio da } \\
\text { vel. aux. }\end{array}$ & $\begin{array}{l}\text { Cálculo do } \\
\text { desvio de } \\
\text { pressão }\end{array}$ & $\begin{array}{l}\text { Cálculo do } \\
\text { desvio de } \\
\text { velocidade }\end{array}$ \\
\hline
\end{tabular}

Figura 7. Divisão percentual do tempo para o cálculo das subrotinas iterativas.

- cálculo da variação da velocidade auxiliar: compreende a montagem das matrizes auxiliares necessárias para a solução da equação da quantidade de movimento e as operações entre elas. Corresponde ao Passo 1 do procedimento apresentado na metodologia pela equação (10).

- cálculo da variação de pressão: compreende a montagem das matrizes auxiliares necessárias para a solução da equação da variação de pressão e as operações entre elas. Corresponde ao Passo 2 do procedimento apresentado na metodologia pela equação (13).

- cálculo da correção da velocidade: compreende a montagem das matrizes auxiliares necessárias para a solução da equação de correção da variação de velocidade e as operações entre elas, atualização dos valores de velocidade e pressão. Corresponde ao Passo 3 do procedimento apresentado na metodologia na equação (16).

Uma análise da figura 7 mostra que a maior parte do tempo é utilizada para a montagem das matrizes globais para os passos 1 e 3 . Portanto, esses passos deveriam ser os principais alvos de qualquer ação no sentido de redução de tempo de processamento. Para uma melhor identificação dos fatores limitantes, os passos 1 e 3 também foram subdivididos. O cálculo do desvio da velocidade auxiliar, Passo 1, que representa 44,9\% da figura 7, está apresentado na figura 8 , e subdividido em: 
- montagem das matrizes: compreende o cálculo e a atualização das matrizes.

- operações entre elas: compreendendo a solução do sistema de equações.

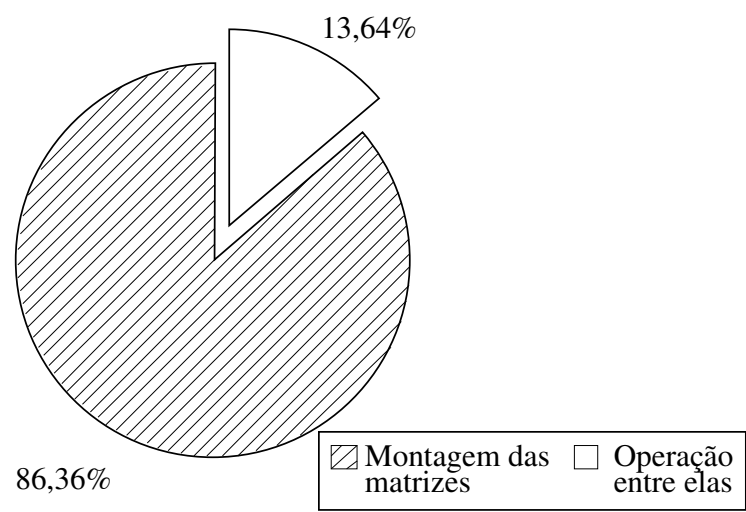

Figura 8. Detalhamento do consumo de tempo para o "Passo 1".

Para este caso apresentado na figura 8 os cálculos e a montagem da matriz global são os principais consumidores de tempo de CPU. Embora, não tenham sido feitos testes adicionais, técnicas alternativas para a montagem da matriz global poderiam ser eficazes na redução do tempo necessário para o processo de solução. O Passo 2, ou a etapa de cálculo do desvio de pressão, que representa $7,7 \%$ da figura 7 , é apresentado na figura 9 , também foi subdividido em:

- montagem das matrizes: compreende o cálculo e a atualização das matrizes.

- operações entre elas: solução da equação.

- cálculo do indicador do desvio de pressão.

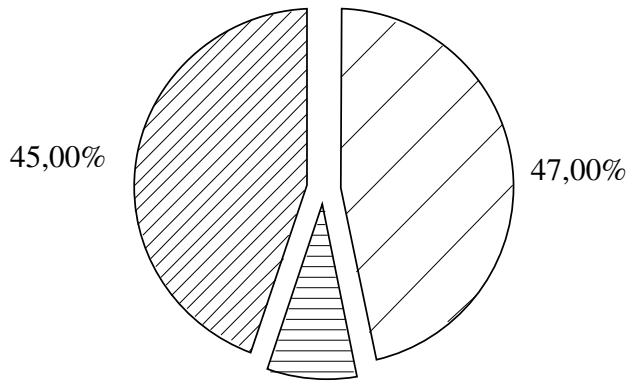

$8,00 \%$

\begin{tabular}{|c|c|}
\hline $\begin{array}{c}\nabla \nexists \text { Montagem } \\
\begin{array}{c}\text { Operação } \\
\text { das matrizes }\end{array} \\
\text { entre elas }\end{array}$ & $\begin{array}{l}\square \text { Cálculo do } \\
\text { desvio de } \\
\text { pressão }\end{array}$ \\
\hline
\end{tabular}

Figura 9. Detalhamento do consumo de tempo para o "Passo 2".
Esta etapa apresenta resultados similares ao do Passo 1, a montagem da matriz é a etapa com maior consumo de tempo. Entretanto, neste passo, também é um grande consumidor de tempo o cálculo do desvio de pressão, que apenas serve para avaliar a convergência do modelo e, por isso, não tem necessidade de ser calculado em todos os intervalos de tempo. Procedimentos de cálculo alternados no tempo desse parâmetro são capazes de reduzir o tempo final necessário para a sua conclusão de maneira significativa.

O cálculo da correção da velocidade, Passo 3, que representa $47,4 \%$ na figura 7, está apresentado na figura 10 e também tem maior parte do tempo gasto na montagem da matriz. Esse tempo, na figura 10, foi dividido em:

- montagem das matrizes: compreende o cálculo e a atualização das matrizes.

- operações entre elas: solução da equação.

- atualização dos valores de velocidade e pressão. Tempo muito pequeno representando menos de $0,01 \%$ do total consumido.

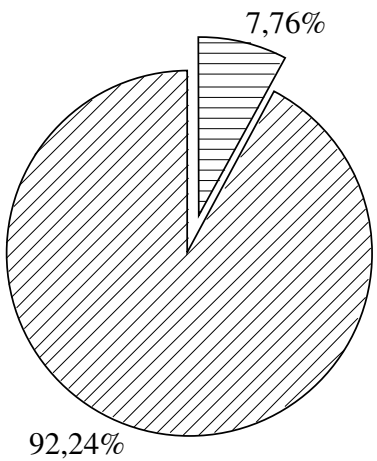

$\square$ Montagem
das matrizes $\begin{aligned} & \text { Operação } \\ & \text { entre elas }\end{aligned} \begin{aligned} & \text { Atulaização } \\ & \text { das } \\ & \text { velocidades }\end{aligned}$

Figura 10. Detalhamento do consumo de tempo para o "Passo 3".

\section{CONCLUSÕES}

A implementação do método por meio do algoritmo mostrou-se plenamente satisfatórios, já que foram alcançados resultados praticamente coincidentes com os disponíveis na literatura.

Além disto, a análise do tempo de cada etapa da solução indicou que a montagem das matrizes é responsável pela maior parte do consumo de tempo de processamento. 
Estudos posteriores devem ser feitos no sentido da utilização de formas alternativas de armazenamento e de atualização das matrizes.

\section{AGRADECIMENTOS}

Os autores agradecem à CAPES pelo suporte financeiro oferecido na forma de bolsa de estudos para o aluno de Pós-Graduação.

\section{REFERÊNCIAS}

[1] N. Massarotti, P. Nithiarasu e O.C. Zienkiewicz. "Characteristic-Based-Split (CBS) Algorithm for Incompressible Flow Problems with Heat Transfer". International Journal of Numerical Methods for Heat \& Fluid Flow. Vol. 8, pp. 969-990. 1998.

[2] O.C. Zienkiewicz, P. Nithiarasu, R. Codina, M. Vazquez e P. Ortiz. "The Characteristic-Based-Split Procedure: A Efficient and Accurate Algorithm for Fluid Problems". Int. J. Numer. Meth. Fluids. Vol. 31, pp. 359-392. 1999.

[3] O.C. Zienkiewicz e R.L. Taylor. "The Finite Element Method". 5 edition. Butterworth-Heinemann. Oxford, England. 2000.

[4] P. Nithiarasu e O.C. Zienkiewicz. "On Stabilization of the CBS Algorithm: Internal and External Time Steps". Int. J. Numer. Meth. Engng. Vol. 48, pp. 875-880. 2000.

[5] P. Nithiarasu e C.B. Liu. "An artificial compressibility based characteristic based split (CBS) scheme for steady and unsteady turbulent incompressible flows". Comput. Methods Appl. Mech. Engrg. Vol. 195, pp. 2961-2982. 2006.

[6] P. Nithiarasu, J.S. Mathur, P. Weatherill e K. Morgan. "Three-dimensional incompressible flow calculations using the characteristic based split (CBS) scheme". International Journal for Numerical Methods in Fluids. Vol. 44, pp. 12071229. 2004.
[7] P. Nithiarasu e O.C. Zienkiewicz. "Analysis of an explicit and matrix free fractional step method for incompressible flows". Comput. Methods Appl. Mech. Engrg. Vol. 195, pp. 5537-5551. 2006.

[8] P. Nithiarasu e O.C. Zienkiewicz. "Analysis of an explicit and matrix free fractional step method for incompressible flows". Comput. Methods Appl. Mech. Engrg. Vol. 195, pp. 5537-5551. 2006.

[9] P. Nithiarasu, N. Massarotti e J.S. Mathur. "Forced convection heat transfer from solder balls on a printed circuit board using the characteristic based split (CBS) scheme". International Journal for Numerical Methods in Heat \& Fluid Flow. Vol. 15, pp. 73-95. 2005.

[10] C.G. Thomas e P. Nithiarasu. "Influences of element size and variable smoothing on inviscid compressible flow solution". International Journal of Numerical Methods for Heat and Fluid Flow. Vol. 15, pp. 420-428. 2005.

[11] U. Ghia, K.N. Ghia e C.T. Shin. "High-Re Solutions for Incompressible Flow Using the Navier-Stokes Equations and a Multigrid Method". Journal of Computational Physics. Vol. 48, pp. 387-411. 1982.

[12] E. Erturk. "Lid Driven Cavity Flow". URLs: http:// www.cavityflow.com. Acesso em: junho/2007.

[13] John W. Eaton. "GNU Octave". Verbatim copying and distribution is permitted in any medium, provided this notice is preserved. University of Wisconsin. Department of Chemical Engineering. Madison WI 53719. Copyright 1998-2006. URLs: http://www.gnu.org/software/octave/. Acesso em: 13 jun. 2007.

[14] P. Nithiarasu. "An efficient artificial compressibility (AC) scheme based on the characteristic based split (CBS) method for incompressible flows". Int. J. for Numer. Meth. Engng. Vol. 56, pp. 18151845. 2003. 